\title{
Diagnostic Accuracy of Coronary Calcium Score Less than 100 in Excluding Coronary Artery Disease
}

\author{
Reza Hanifehpour, ${ }^{1, *}$ Marzieh Motevalli, ${ }^{1,2}$ Hossein Ghanaati, ${ }^{1}$ Mona Shahriari, ${ }^{1}$ and Mounes Aliyari \\ Ghasabeh $^{1}$ \\ ${ }^{1}$ Advanced Diagnostic and Interventional Radiology Research Center (ADIR), Tehran University of Medical Sciences, Tehran, Iran \\ ${ }^{2}$ Department of Radiology, Rajaei Cardiovascular Medical and Research Center, Iran University of Medical Sciences, Tehran, Iran \\ "Corresponding author: Reza Hanifehpour, Advanced Diagnostic and Interventional Radiology Research Center, Medical Imaging Center, Tehran, Iran. Tel: +98-9143912450, Fax: \\ +98-2166945117, E-mail: hanifehr@yahoo.com
}

Received 2013 December 09; Revised 2014 September 14; Accepted 2014 September 17.

\begin{abstract}
Background: Coronary artery calcium score (CACS) is a quantitative assessment of calcifications and an established predictor of cardiovascular events.

Objectives: In this study, we evaluated the diagnostic accuracy, negative predictive value (NPV), positive predictive value (PPV), specificity and sensitivity of CACS less than 100 in predicting significant coronary artery stenosis in patients with risk of coronary artery disease $(\mathrm{CAD})$ in a vessel-based analysis.

Patients and Methods: A cross sectional study was carried out on a study population of 2527 consecutive stable patients with symptoms suggestive of CAD who were referred for coronary computed tomographic angiography (CCTA). We performed 1343 studies with 256 slice machine in Shahid Rajaee hospital and the other studies were carried out with 64 slice machine in Imam Khomeini hospital and the calcium score was quantified according to the Agatston method.

Results: At the cutoff point of 100 for coronary calcium scoring, there was high specificity (87\%), high sensitivity (79\%), high efficiency (84\%), high PPV (79\%), and high NPV (87\%) in the diagnosis of significant stenosis in the whole heart. The frequency of zero calcium scoring was $59 \%$ in normal or nonsignificant stenosis and $7.6 \%$ in significant stenosis in the whole heart. Calcium scoring increased with greater severity of the arterial stenosis (P values $<0.001$ ).

Conclusion: We conclude that coronary calcium scoring provided useful information in the management of patients. In CACS less than 100 , it has a NPV of $87 \%$ in excluding significant stenosis in patients with the risk of CAD but it does not have enough diagnostic accuracy for surely excluding coronary stenosis, so we should perform a combination of CACS and coronary CT angiography for patients.
\end{abstract}

Keywords: Coronary Artery Disease, Multidetector Computed Tomography, Calcium Score, Diagnostic Efficacy

\section{Background}

Coronary artery disease (CAD) is the most common cause of heart attack and death in the world (1). Since CAD imposes a high economic burden to the health system, it is important to identify the best accurate test for diagnosing CAD in order to reduce time and cost consumption.

Recently, coronary computed tomographic angiography (CCTA) which is a noninvasive imaging method with high diagnostic accuracy (2) is used for detecting coronary artery stenosis. Coronary artery calcium (CAC) which is a good marker of atherosclerosis is evaluated at the beginning of CCTA with less radiation and it is an established predictor of cardiovascular events (3-6). Most frequently, the Agatston score has been used to measure the total coronary calcified plaque (7). Therefore, if we demonstrate that the coronary artery calcium score (CACS) has a high diagnostic accuracy in diagnosing and excluding coronary artery disease, we can prevent unnecessary critical and invasive interventions in the treatment of patients suspicious of CAD.

\section{Objectives}

The aim of this study was to evaluate the diagnostic accuracy, negative predictive value (NPV), positive predictive value (PPV), specificity, and sensitivity of CACS less than 100 in predicting coronary artery stenosis in patients with a low to intermediate risk of CAD in a vessel-based analysis.

\section{Patients and Methods}

\subsection{Patient Enrollment}

This was a cross sectional study carried out in Tehran, Iran among consecutive stable patients with signs and 
symptoms suggestive of CAD who were referred to Shahid Rajaee and Imam Khomeini cardiology center between January 2008 and May 2013. After obtaining written informed consent from the patients, 2527 patients underwent CCTA and calcium scoring. The study design was approved by the institutional review committee of hospitals. Exclusion criteria were previous coronary artery stent placement, increased serum creatinine level, and an allergic response to intravenous contrast materials.

\subsection{CT Protocol and Image Acquisition}

One thousand three hundred forty three patients were scanned on a dual source, 128-slices (256 data output channels) multi detector CT scan (Somatom definition flash, Siemens medical solutions, Syngo CT2011A, Germany, 2009) in Shahid Rajaee hospital and the others were scanned on a GE 64 multi-slice CT scan (GE Lightspeed VCT 64 slice CT scanner, USA, 2006) in Imam Khomeini hospital. Patients who were on a beta-blocker continued their medication but the other patients did not receive a beta-blocker to decrease their heart rate (the 128-slice dual source scanner is a very fast scanner that can provide high quality images in a patient with high or irregular heart rate and it is not necessary to prescribe a beta-blocker before scan to control the heart rate). Before coronary CT angiography, CAC scoring was performed with non overlapping images with a slice thickness of $3 \mathrm{~mm}$ and medium sharp convolution Kernel (B35f). Following calcium scoring, all patients received a single puff of nitroglycerin 0.4 $\mathrm{mg}$ (GLYTRIN $0.4 \mathrm{mg} /$ dose, Sanofi, Moreton Wirral, UK). After 2 minutes, a bolus of $80 \mathrm{~mL}$ of iohexol (Omnipaque 350, $350 \mathrm{mg} / \mathrm{mL}$, GE Healthcare, Ireland cork, Ireland) followed by $30 \mathrm{~mL}$ saline chaser, was injected into an antecubital vein via a catheter (injection rate 4 - $6 \mathrm{~mL} /$ second according to venous catheter gauge) (6). Contrast-agent application was controlled by bolus-tracking in the aortic root. CT angiography and image acquisition started $10 \mathrm{~s}$ after the signal attenuation reached the predefined threshold of 130 Hounsfield units (HU). ECG-pulsing for radiation dose reduction was applied in all patients. With two tubes and two detectors mounted at orthogonal orientation in the gantry, the transmitted data required for the reconstruction of one image slab could be acquired in $3-5$ seconds breath holding.

Axial images were reconstructed with $0.75 \mathrm{~mm}$ slice thickness and using medium soft tissue Kernel (B26f) and retrospective ECG gating. The reconstructions were performed in best of diastole and systole for using circulation application.

\subsection{Image Analysis}

For image analysis, the coronary artery tree was separated into 18 segments (within four main coronary artery vessels: LM, left main; LAD, left anterior descending artery; RCA, right coronary artery; and LCX, left circumflex artery) according to the society of cardiovascular computed tomography (SCCT) guidelines. Each segment diameter was $>1.5 \mathrm{~mm}$. For assessment of stenosis severity, we used recommended quantitative stenosis grading with a little modification. Recommended quantitative stenosis grading defines coronary stenosis severity as: 0 , normal: absence of plaque and no luminal stenosis; 1, minimal: plaque with $<25 \%$ stenosis; 2 , mild: 25 - 49\% stenosis; 3 , moderate: 50 - $69 \%$ stenosis; 4 , severe: 70 - 99\% stenosis; and 5, occluded. We modified this grading to: 0 , normal: absence of plaque and no luminal stenosis; 1, mild: 1 - 49\% stenosis; 2, moderate: 50 - 69\% stenosis; 3, severe: 70 - 99\% stenosis; 4 , occluded. In addition, for evaluation of obstruction in each of the four coronary arteries, three cut off points were described: at least one segment with any kind of stenosis (0), at least one segment with $\geq 50 \%$ stenosis ( $\geq 50$ ), and at least one segment with $\geq 70 \%$ stenosis ( $\geq$ $70)$.

CAC scores which identify as distribution of imaging pixels with calcification density $>130 \mathrm{HU}$ were calculated in each coronary artery (LM, LAD, RCA, and LCX) by using the Agatston score method. The total coronary artery calcium score was also calculated (7).

Each patient with coronary CT angiogram was evaluated by two experienced cardio thoracic interventional radiologists who were unaware of the clinical history of the patients. Consensus was obtained by consultation on cases with different reports.

\subsection{Statistical Analysis}

Statistical analysis was performed using SPSS for windows, version 16.0 (SPSS Inc. Chicago, IL, USA). We used oneway ANOVA test to obtain mean values of calcium scoring for each artery.

\section{Results}

Totally, 2527 patients underwent cardiac CT angiography between January 2008 and May 2013. Among them, 1343 studies were carried out with 256 slice machine in Rajayee hospital and the others were performed with 64 slice machine in Imam Khomeini hospital.

The mean age of the patients was $57 \pm 12.6$ (7-93) years old. Totally, 1458 (58.8\%) of the patients were male. 
In all patients, the degree of stenosis in each main artery (LM, LAD, LCX, and RCA) was determined. In addition, the total calcium scoring and calcium scoring in four mentioned main branches was determined.

Presence and degree of stenosis in different portions of LAD, LCX, RCA, and LM were determined (Table 1).

Considering the maximum severity stenosis in each artery, we determined the whole situation of LAD, LCX, and RCA. For example, if the proximal portion of LAD had moderate stenosis and the other parts had mild stenosis or were normal, the whole LAD was labeled as moderate stenosis. Accordingly, the frequency of different severity stenosis in each artery was determined (Table 2). Different severities of LM are mentioned in Table 1.

Clinically significant stenosis in the LM was considered as a stenosis greater than $50 \%$, while it was considered in other arteries when the stenosis was greater than $70 \%$. In addition, we combined the data of all arteries and determined the stenosis in the whole heart (Table 2).

On the other hand, we determined the mean calcium scoring of each artery separately and compared it among different patients categorized according to maximum severity. In ANOVA tests performed for comparison of the calcium scores, it was shown that the calcium scoring is increased with greater severity of the arterial stenosis (all P values < 0.001) (Table 3 ).

In $\mathrm{LAD}$, the frequency of zero calcium scoring in normal or non-significant stenosis was 91.6\% (2152/2349) while this frequency was $63.1 \%$ in LM significant stenosis (111/176) $(\mathrm{P}<0.001)$.

This profile was significant in LAD, LCX, RCA, and the whole heart too (all P values $<0.001$ ) (Table 4 ).

The frequency of zero calcium scoring in LM, LAD, LCX, RCA, and the whole heart was $89.6 \%, 44 \%, 62.4 \%, 59.4 \%$, and $39.8 \%$, respectively.

Considering a cut off point of 100 for calcium scoring, we determined the diagnostic efficacies of this measurement for diagnosis of significant stenosis in the whole heart.

The result has been mentioned as follows:

Whole heart: sensitivity $=0.7911$ ( $95 \%$ confidence interval [CI]: 0.7637-0.8166); specificity $=0.8780$ (95\% CI: 0.8609 $0.8937)$; efficiency (correct classification rate) $=0.8455(95 \%$ CI: 0.8309 - 0.8594); PPV = 0.7945 (95\% CI: 0.7672 - 0.8199); $\mathrm{NPV}=0.8758$ (95\% CI: 0.8585 - 0.8916); positive likelihood ratio $(\mathrm{PLR})=6.4845$ (95\% CI: 5.6588 - 7.4307); negative likelihood ratio $(\mathrm{NLR})=4.2028$ (95\% CI: 3.7069 - 4.7651).

\section{Discussion}

The main finding of our study is that at the cutoff point of 100 for coronary calcium scoring we have a high speci- ficity (87\%), high sensitivity (79\%), high efficiency (84\%), high PPV (79\%), and high NPV (87\%) in the diagnosis of significant stenosis in the whole heart. In our study, the frequency of zero calcium scoring in normal or nonsignificant stenosis and significant stenosis in the whole heart was 59\% and 7.6\%, respectively. Also, it was shown that the calcium scoring increased with greater severity of the arterial stenosis (Pvalues < 0.001).

Recently, the performance of CACS for diagnosing stenosis in coronary arteries has been discussed widely. Many studies emphasize that higher CACS is associated with progressive atherosclerosis and an increased prevalence of coronary artery stenosis (3, 8-11), similar to the results in our study.

In our study, we found that at the cutoff point of 100 , we have high specificity (87\%), sensitivity (79\%), efficiency (84\%), PPV (79\%), and high NPV (87\%) for diagnosing significant stenosis and excluding CAD. Despite this high NPV we cannot surely exclude significant coronary stenosis in patients with CACS less than 100.

In our study, the frequency of zero calcium scoring in normal or non-significant stenosis in LM, LAD, LCX, RCA, and the whole heart was $91.6 \%, 61.8 \%, 72.7 \%, 74.3 \%$, and $59 \%$, respectively. In significant stenosis, it was $63.1 \%, 8.1 \%, 18.4 \%$, $14.1 \%$, and $7.6 \%$, respectively. LM artery plaques are less calcified, so we have a higher percentage of significant stenosis among patients with zero CACS. Some studies reported that a calcium score of zero can rule out significant stenosis (12-16), but in our study, we cannot surely exclude significant stenosis in patients with a CACS of zero or less than 100. Our results were consistent with other studies which concluded that although CACS is a good marker of atherosclerosis and it is an established predictor of cardiovascular events (3-5), CACS of zero does not exclude obstructive stenosis (10) and CCTA is highly superior than a CACS in CAD detection (17). There are some differences in results between these studies that can be due to differences in measurement techniques, equipment, patient classification, diffuse non significant calcium burden, and complicated plaques.

Our study was limited because of time and cost restriction. Our gold standard for evaluating CACS for diagnosing stenosis was CCTA instead of invasive coronary angiography, which is the gold standard method of detection of CAD. On the other hand, different studies have shown that 64-slice multi detector CT scan, which is the most usual scanner used for CAD detection, has 90 - 94\% sensitivity and $96-100 \% \operatorname{NPV}(16,18,19)$ and a more advanced device such as 128-slice CT has a higher detection rate (20).

We conclude that coronary calcium scoring provided useful information. In CACS less than 100, it has a negative predictive value of $87 \%$ in excluding significant stenosis in 
Table 1. Presence and Severity of Stenosis in Different Parts of Coronary Arteries ${ }^{\mathrm{a}}$

\begin{tabular}{|c|c|c|c|c|c|c|}
\hline & & Normal & Mild Stenosis (1 - 50\%) & Moderate Stenosis (51 - 70\%) & Severe Stenosis (71 - 99\%) & Complete Occlusion (100\%) \\
\hline LM & & $2089(82.7)$ & $262(10.4)$ & $65(2.6)$ & $105(4.2)$ & $6(0.2)$ \\
\hline \multicolumn{7}{|l|}{ LAD } \\
\hline & Proximal portion & $1322(52.3)$ & $375(14.8)$ & $238(9.4)$ & $444(17.6)$ & $148(5.9)$ \\
\hline & Mid portion & 145957.7() & $247(9.8)$ & $204(8.1)$ & $427(16.9)$ & $190(7.5)$ \\
\hline & Distal portion & $2241(88.7)$ & $99(3.9)$ & $48(1.9)$ & $90(3.6)$ & $49(1.9)$ \\
\hline \multicolumn{7}{|l|}{ LCX } \\
\hline & Proximal portion & $1787(70.7)$ & $273(10.8)$ & $128(5.1)$ & $268(10.6)$ & $71(2.8)$ \\
\hline & Mid portion & $2114(83.7)$ & $109(4.3)$ & 68() 2.7 & $159(6.3)$ & $77(3)$ \\
\hline & Distal portion & $2384(94.3)$ & $32(1.3)$ & $27(1.1)$ & $50(2)$ & $34(1.3)$ \\
\hline \multicolumn{7}{|l|}{ RCA } \\
\hline & Proximal portion & $1769(70)$ & $294(11.6)$ & $99(3.9)$ & $266(10.5)$ & $99(3.9)$ \\
\hline & Mid portion & $1840(72.8)$ & $169(6.7)$ & $110(4.4)$ & $233(9.2)$ & $175(6.9)$ \\
\hline & Distal portion & $2069(81.9)$ & $111(4.4)$ & $70(2.8)$ & $170(6.7)$ & $107(4.2)$ \\
\hline
\end{tabular}

Abbreviations: LAD, left anterior descending; LCX, left circumflex; LM, left main; RCA, right coronary artery.

${ }^{\mathrm{a}}$ Values are expressed as No. (\%).

Table 2. Frequency of Stenosis and Clinically Significant Stenosis in Each Coronary Artery ${ }^{\mathrm{a}}$

\begin{tabular}{|c|c|c|c|c|c|}
\hline & Normal & Mild Stenosis (1 - 50\%) & Moderate Stenosis (51 - 70\%) & Severe Stenosis (71 - 99\%) & Complete Occlusion (100\%) \\
\hline LM & $2089(82.7)$ & $262(10.4)$ & $65(2.6)$ & $105(4.2)$ & $6(0.2)$ \\
\hline LAD & $1057(41.8)$ & $330(13.1)$ & $308(12.2)$ & $527(20.9)$ & $305(12.1)$ \\
\hline LCX & $1622(64.2)$ & $265(10.5)$ & $166(6.6)$ & $339(13.4)$ & $135(5.3)$ \\
\hline RCA & $1503(59.5)$ & $266(10.5)$ & $135(5.3)$ & $345(13.7)$ & $278(11)$ \\
\hline Whole heart & $349(13.8)$ & $287(11.4)$ & $461(18.2)$ & $483(19.1)$ & $947(37.5)$ \\
\hline
\end{tabular}

Abbreviations: LAD, left anterior descending; LCX, left circumflex; LM, left main; RCA, right coronary artery.

${ }^{a}$ Values are expressed as No. (\%)

Table 3. Mean Calcium Scoring in Different Severities of Stenosis in Each Coronary Artery ${ }^{\mathrm{a}}$

\begin{tabular}{|c|c|c|c|c|c|c|}
\hline & Normal & $\begin{array}{l}\text { Mild Stenosis } \\
\qquad(\mathbf{1 - 5 0} \%)\end{array}$ & $\begin{array}{c}\text { Moderate Stenosis } \\
\qquad(51-70 \%)\end{array}$ & $\begin{array}{l}\text { Severe Stenosis } \\
\qquad(71-99 \%)\end{array}$ & Complete Occlusion (100\%) & PValue \\
\hline LM calcium scoring & $2.9 \pm 23.3$ & $16.4 \pm 46.3$ & $23.2 \pm 62.7$ & $50.1 \pm 93$ & $21.1 \pm 48.5$ & $<0.0001$ \\
\hline LAD calcium & $13.5 \pm 128$ & $81.6 \pm 282.6$ & $135.3 \pm 216.2$ & $311.6 \pm 351.2$ & $375.3 \pm 444.6$ & $<0.0001$ \\
\hline LCx calcium & $12.2 \pm 111.3$ & $54.2 \pm 117.4$ & $101.6 \pm 127$ & $130.5 \pm 225$ & $144.3 \pm 199.5$ & $<0.0001$ \\
\hline RCA calcium & $10.9 \pm 108.8$ & $91.6 \pm 258.8$ & $152 \pm 232.5$ & $297.5 \pm 499.6$ & $237.3 \pm 319.3$ & $<0.0001$ \\
\hline Whole heart calcium scoring & $21.8 \pm 372.7$ & $105.4 \pm 433$ & $218.4 \pm 439.3$ & $537.9 \pm 740.8$ & $736.2 \pm 723.4$ & $<0.0001$ \\
\hline
\end{tabular}

Abbreviations: LAD, left anterior descending; LCX, left circumflex; LM, left main; RCA, right coronary artery; SD, standard deviation.

${ }^{\mathrm{a}}$ Values are expressed as mean $\pm \mathrm{SD}$.

patients with a risk of CAD, but it does not have enough diangiography in combination for patients. agnostic accuracy for excluding coronary stenosis. Therefore, we should perform both CAC scoring and coronary CT 
Table 4. Frequency of Zero Calcium Scoring in Normal or Non-Significant Stenosis in Each Aarteries and the Whole Heart

\begin{tabular}{|c|c|c|}
\hline & Zero Calcium Score, \% & PValue \\
\hline LM calcium scoring & & $<0.0001$ \\
\hline $\begin{array}{l}\text { Normal or non significant } \\
\text { stenosis }\end{array}$ & 91.60 & \\
\hline Significant Stenosis & 63.10 & \\
\hline LAD calcium scoring & & $<0.0001$ \\
\hline $\begin{array}{l}\text { Normal or non significant } \\
\text { stenosis }\end{array}$ & 61.80 & \\
\hline Significant Stenosis & 8.10 & \\
\hline LCX calcium scoring & & $<0.0001$ \\
\hline $\begin{array}{l}\text { Normal or non significant } \\
\text { stenosis }\end{array}$ & 72.70 & \\
\hline Significant Stenosis & 18.40 & \\
\hline RCA calcium scoring & & $<0.0001$ \\
\hline $\begin{array}{l}\text { Normal or non significant } \\
\text { stenosis }\end{array}$ & 74.30 & \\
\hline Significant Stenosis & 14.10 & \\
\hline Whole Heart calcium scoring & & $<0.0001$ \\
\hline $\begin{array}{l}\text { Normal or non significant } \\
\text { stenosis }\end{array}$ & 59 & \\
\hline Significant Stenosis & 7.60 & \\
\hline
\end{tabular}

Abbreviations: LAD, left anterior descending; LCX, left circumflex; LM, left main RCA, right coronary artery.

\section{Footnotes}

Authors' Contributions: Study concept and design was performed by Reza Hanifehpour, Marzieh Motevalli, Mona Shahriari, and Mounes Aliyari Ghasabeh. Analysis and interpretation of data was carried out by Mona Shahriari, and Mounes Aliyari Ghasabeh. Drafting of the manuscript was done by Mona Shahriari, Mounes Aliyari Ghasabeh, and Reza Hanifehpour. Critical revision of the manuscript for important intellectual content was performed by Marzieh Motevalli, and Hussein Ghanaati. Statistical analysis was carried out by Mona Shahriari, and Mounes Aliyari Ghasabeh. Also, Marzieh Motevalli developed the original idea.

Financial Disclosure: We have no financial interests related to the manuscript.

Funding/Support: No funding/support was received.

\section{References}

1. Castelli WP. Epidemiology of coronary heart disease: the Framingham study. Am J Med. 1984;76(2a):4-12. [PubMed: 6702862]

2. Sun Z, Jiang W. Diagnostic value of multislice computed tomography angiography in coronary artery disease: a meta-analysis. Eur J Radiol. 2006;60(2):279-86. doi: 10.1016/j.ejrad.2006.06.009. [PubMed: 16887313].
3. Budoff MJ, Shaw LJ, Liu ST, Weinstein SR, Mosler TP, Tseng PH, et al. Long-term prognosis associated with coronary calcification: observations from a registry of 25,253 patients. J Am Coll Cardiol. 2007;49(18):1860-70. doi: 10.1016/j.jacc.2006.10.079. [PubMed: 17481445].

4. Chen LC, Ding PY, Chen JW, Wu MH, Liu JC, Lan GY, et al. Coronary artery calcium determined by electron beam computed tomography for predicting angiographic coronary artery disease in moderateto high-risk Chinese patients. Cardiology. 2001;95(4):183-9. [PubMed: 11585993].

5. Vliegenthart R, Oudkerk M, Hofman A, Oei HH, van Dijck W, van Rooij FJ, et al. Coronary calcification improves cardiovascular risk prediction in the elderly. Circulation. 2005;112(4):572-7. [PubMed: 16009800].

6. Liu Q, Qian Z, Marvasty I, Rinehart S, Voros S, Metaxas DN. Lesionspecific coronary artery calcium quantification for predicting cardiac event with multiple instance support vector machines. Med Image Comput Comput Assist Interv. 2010;13(Pt 1):484-92. [PubMed: 20879266].

7. Agatston AS, Janowitz WR, Hildner FJ, Zusmer NR, Viamonte MJ, Detrano R. Quantification of coronary artery calcium using ultrafast computed tomography.J Am Coll Cardiol. 1990;15(4):827-32. [PubMed: 2407762].

8. Detrano R, Guerci AD, Carr JJ, Bild DE, Burke G, Folsom AR, et al. Coronary calcium as a predictor of coronary events in four racial or ethnic groups. N Engl J Med. 2008;358(13):1336-45. doi: 10.1056/NEJMoa072100. [PubMed: 18367736].

9. Nasir K, Shaw LJ, Liu ST, Weinstein SR, Mosler TR, Flores PR, et al. Ethnic differences in the prognostic value of coronary artery calcification for all-cause mortality. J Am Coll Cardiol. 2007;50(10):953-60. doi: 10.1016/j.jacc.2007.03.066. [PubMed: 17765122].

10. Gottlieb I, Miller JM, Arbab-Zadeh A, Dewey M, Clouse ME, Sara L, et al. The absence of coronary calcification does not exclude obstructive coronary artery disease or the need for revascularization in patients referred for conventional coronary angiography. J Am Coll Cardiol. 2010;55(7):627-34. doi: 10.1016/j.jacc.2009.07.072. [PubMed: 20170786].

11. Motevalli M, Ghanaati H, Firouznia K, Kargar J, Aliyari Ghasabeh M, et al. Diagnostic efficacy of vessel specific coronary calcium score in detection of coronary artery stenosis. Iran Red Crescent Med J. 20014;16(12):e26010. doi: 10.5812/ircmj.26010. [PubMed: 25763246].

12. Mouden M, Timmer JR, Reiffers S, Oostdijk AH, Knollema S, Ottervanger JP, et al. Coronary artery calcium scoring to exclude flowlimiting coronary artery disease in symptomatic stable patients at low or intermediate risk. Radiology. 2013;269(1):77-83. doi: 10.1148/radiol.13122529. [PubMed: 23788718].

13. Sosnowski M, Pysz P, Szymanski L, Gola A, Tendera M. Negative calcium score and the presence of obstructive coronary lesions in patients with intermediate CAD probability. Int JCardiol. 2011;148(1):e168. doi: 10.1016/j.ijcard.2009.01.077. [PubMed:19324431].

14. Meyer M, Henzler T, Fink C, Vliegenthart R, Barraza JJ, Nance JJ, et al. Impact of coronary calcium score on the prevalence of coronary artery stenosis on dual source CT coronary angiography in caucasian patients with an intermediate risk. Acad Radiol. 2012;19(11):1316-23. doi: 10.1016/j.acra.2012.06.006. [PubMed: 22897947].

15. Konieczynska M, Tracz W, Pasowicz M, Przewlocki T. Use of coronary calcium score in the assessment of atherosclerotic lesions in coronary arteries. Kardiol Pol. 2006;64(10):1073-9. [PubMed:17089239] discussion 1080-1.

16. Cademartiri F, Maffei E, Palumbo A, Martini C, Seitun S, Tedeschi C, et al. Diagnostic accuracy of computed tomography coronary angiography in patients with a zero calcium score. Eur Radiol. 2010;20(1):81-7. doi: 10.1007/s00330-009-1529-9. [PubMed: 19657651]. 
17. Hadamitzky M, Distler R, Meyer T, Hein F, Kastrati A, Martinoff S, et al. Prognostic value of coronary computed tomographic angiography in comparison with calcium scoring and clinical risk scores. Circ Cardiovasc Imaging. 2011;4(1):16-23. doi: 10.1161/CIRCIMAGING.110.955351. [PubMed: 20884832].

18. Stein PD, Yaekoub AY, Matta F, Sostman HD. 64-slice CT for diagnosis of coronary artery disease: a systematic review. Am J Med 2008;121(8):715-25. doi: 10.1016/j.amjmed.2008.02.039. [PubMed:
18691486].

19. Liew GY, Feneley MP, Worthley SG. Appropriate indications for computed tomography coronary angiography. Med J Aust. 2012;196(4):246-9. [PubMed: 22409689].

20. Achenbach S, Marwan M, Schepis T, Pflederer T, Bruder H, Allmendinger T, et al. High-pitch spiral acquisition: a new scan mode for coronary CT angiography. J Cardiovasc Comput Tomogr. 2009;3(2):11721. doi: 10.1016/j.jcct.2009.02.008. [PubMed: 19332343]. 